\title{
Traffic Engineering enforcement in multi-domain SDN orchestration of Multi-Layer (packet/optical) networks
}

\author{
A. Mayoral(1), R. Vilalta(1), R. Casellas ${ }^{(1)}$, R. Muñoz ${ }^{(1)}$, R. Martínez $^{(1)}$ \\ (1) CTTC, Av. Carl Friedrich Gauss 7, Castelldefels, 08860, Spain, arturo.mayoral@cttc.es
}

\begin{abstract}
We introduce an SDN orchestration architecture to enable the introduction of E2E TE policies in a multi-domain, multi-layer network scenario. The ABNO is used as reference architecture for the SDN orchestration of packet/optical SDN controllers using Flow Service Classification.
\end{abstract}

\section{Introduction}

Introducing automation and dynamicity on the provisioning and release of multi-layer and multidomain end-to-end (E2E) connectivity services is a major concern of network operators in order to increase the revenues of their deployed network infrastructure. Software Defined Networking (SDN) has appeared as a new networking paradigm to drastically reduce the cost of operation and reduce the time-to-market of introducing a new service, by increasing the level of automation on the control and configuration of the network infrastructure. The need of assure the Return-Of-Investment (Rol) of existing network infrastructure suggests in the mid-term an hybrid scenario where traditional control and management systems, such Generalized Multiprotocol Label Switching (GMPLS), will coexist with the emerging SDN. Therefore, we can assume a network comprised by multiple control instances which need to be orchestrated in order to achieve the so-desired dynamicity on the management and control of E2E services.

Network infrastructure monetization depends of efficient control of the traffic flow. Traditionally, this has been achieved by the introduction of Traffic Engineering (TE) policies to take benefit of highly optimized routing algorithms which fully exploit the network capacity. In SDN, the TEenabler mechanisms have been not fully researched, neither the impact that TE policies can introduce into the jointly orchestration of multiple network domains comprising different transport technologies. For instance, in OpenFlow $^{1}$ (OF)-based SDN deployments, traffic differentiation and traffic rate limitation were not enabled until 1.3 version.

Based on the IETF proposed Applicationbased Network Operations (ABNO) ${ }^{2}$, an SDN orchestration architecture (Fig.1) is introduced to provide E2E connectivity services in a multidomain, multi-vendor and multi-layer network scenario. An experimental validation of the E2E provisioning of connection across multiple domains comprising SDN/OF and GMPLS control planes was already demonstrated in a previous work ${ }^{3}$. In the present paper, we focus on addressing the enforcement of TE metrics at the orchestration layer, with two principal objectives: (1) Multi-Layer E2E traffic aggregation; and (2) E2E Quality-of-Service (QoS) enabling. The three major challenges which must be solved by the orchestration architecture are: a) the multilayer and multi-domain topology management; $b$ ) the end-to-end, TE-aware path computation; and c) the QoS guarantee into the E2E service provisioning through different provisioning interfaces, using per-flow service classification.

\section{TE-aware SDN orchestration architecture}

To fully exploit the network utilization, TEstrategies should be applied to place the traffic where the network resources are available. Our proposed SDN orchestration architecture ${ }^{4}$, has been extended by introducing TE parameters (bandwidth, delay sensitivity, TE metric, etc.) into the network topologies managed by orchestration layer. Specifically, in the proposed architecture, the Topology Manager (TM) is the responsible of the Traffic Engineering Database (TED) management, where the multilayer, multi-domain topologies are stored. The TE enforcement strategy consist in the proactive reservation of the network capacity and its dynamic representation into the TED network topologies. The relevant topological parameters necessary to include at orchestration layer TED are: the maximum reservable bandwidth and the unreserved bandwidth. The former is obtained from the topology information retrieved by the per-domain controllers and the latter must be dynamically updated after every new connection is effectively established.

A multilayer PCE using the aforementioned TED, is included within the ABNO to perform the E2E path computations. In a previous work ${ }^{5}$, a Constrained Shortest Path First (CSPF) based algorithm which uses the delay as a TE-metric was presented. In our proposal, the unreserved bandwidth on every link is taken as a constraint by the algorithm to calculate the E2E path across 

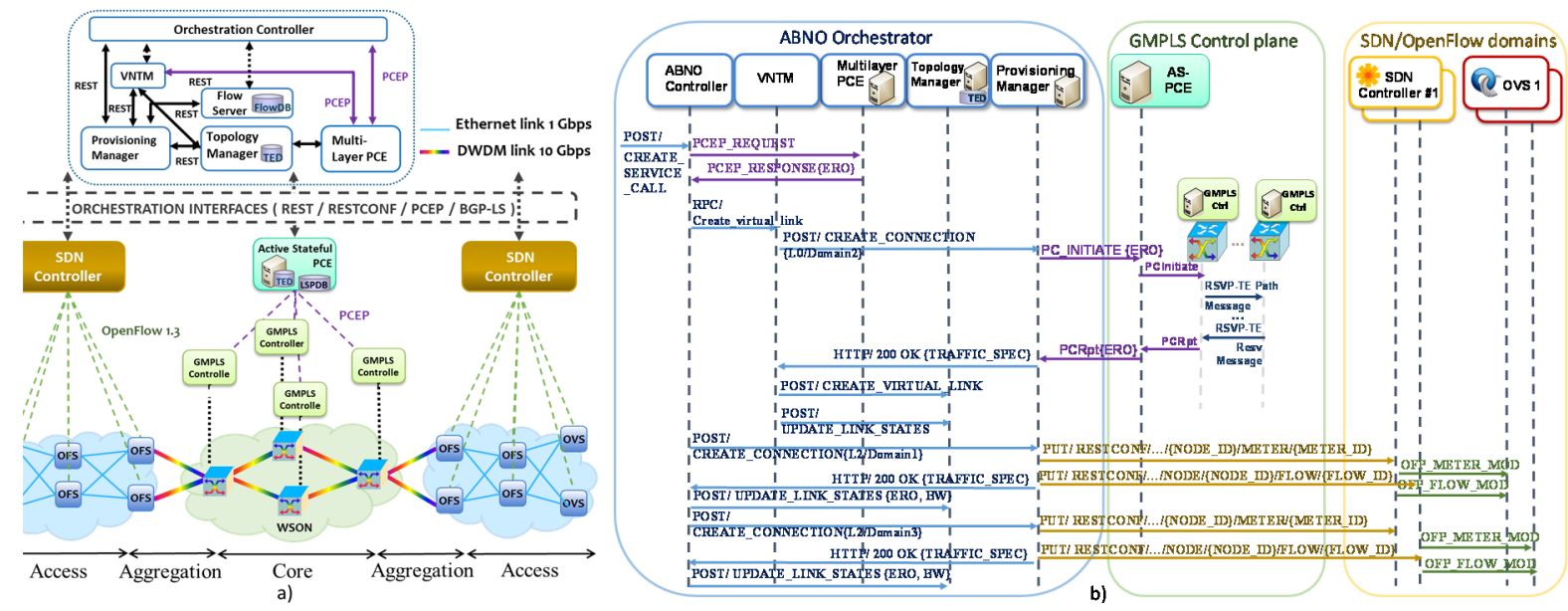

Fig. 1: a) Reference SDN orchestration architecture; b) Multilayer E2E service orchestration workflow.

the multi-layer network. The CSPF output returned within the PCEP Response includes a multilayer Explicit Route Object (ERO) representing the path combining packet links (physical or virtual) and/or optical hops where the layer adaptation is ensured. Optical segments are subject to the Wavelength Continuity Constraint (WCC) and the optical wavelength is included in the ERO if the E2E path traverses a Wavelength Switched Optical Network (WSON) network segment.

An E2E service may trigger the provisioning of one or more lower-layer connections depending on the need for transport the traffic through. The reservation of lower-layer resources (i.e. an optical circuit) must be advertised into the higherlayer topology as a new logical Virtual Link (VLink). The Virtual Network Topology Manager (VNTM) is the ABNO component responsible of the management of Vlink creation, which involves the provisioning of lower-layer connections and the creation of the logical representations into the multilayer topology. When a new VLink is created, it is characterized with the TE information of the underlying connection (i.e. total available bandwidth, aggregated delay).

\section{Bandwidth reservation in SDN orchestration}

TE-aware traffic aggregation requires the guarantee of the effective bandwidth reservation into the E2E service provisioning. While in a WSON GMPLS domain a fixed amount of physical resources $(50 \mathrm{GHz}$ wavelength) are reserved for each Label Switched Path (LSP), in a packet-based OF domains it is necessary to limit the bandwidth assigned to each flow (flow service classification) in order to preserve a certain QoS (i.e. bandwidth) assured to each E2E services. OF v1.3 introduces a new message (i.e. OFPT_METER_MOD) which enables the specification of traffic meters into the OFswitches, with an associated Band-rate and a
QoS-enabling strategy, such dropping packets at a determined Drop-rate or Differentiated Services Code Point (DSCP) packet tagging to allow DiffServ. Flows can be attached to these predefined meters, associating a maximum rate to each flow. A Meter instruction has to be included inside the OFPT_FLOW_MOD message indicating the Meter-ld desired to be attached to.

Fig.1.b. shows a complete E2E service orchestration workflow including the effective bandwidth reservation into the packet domains employing OFv1.3. After every connection is established, the Traffic Specification containing the Peak Rate of the connection is returned to the orchestrator and the affected links are updated with the certain amount of network capacity that has been reserved.

\section{Experimental validation}

The proposed architecture has been experimentally validated in the ADRENALINE Testbed. The network scenario consists of two packet SDN/OpenFlow domains based on Ethernet transport technology and an aggregation optical transport network. Each packet domain consists of four OF Switches deployed using standard Custom Off The Shelf (COTS) hardware and running OpenVSwitch (OVS) and Lagopus, which are all controlled by a per-domain SDN controller (based on OpenDaylight) using OF 1.0 and 1.3. Each OF border switch has been deployed into COTS hardware, a $10 \mathrm{~Gb} / \mathrm{s}$ XFP tunable transponder and OVS technology. The aggregation network consists into a GMPLS-controlled WSON centrally managed by an Active-Stateful PCE (AS-PCE). The optical network infrastructure consists on: 2 ROADMs and 2 OXCs providing reconfigurable E2E lightpaths, deploying a total of $610 \mathrm{~km}$ of G.652 and G.655 optical fiber, with six DWDM wavelengths per optical link. 
Fig.3. shows the traffic capture of the control overhead of the E2E orchestration of a single SERVICE_CALL request, demanding a $100 \mathrm{Mbps}$ Ethernet (L2) service. Firstly, the capture validates the multilayer orchestration by showing a virtual link creation supported by a bidirectional optical LSP requested to an AS-PCE (PCEP Initiate and Report messages). Secondly, the UPDATE_LINK_STATE message is shown, which is the responsible of update the link states after a connection (at any layer) is created. Finally, the per-flow traffic limitation in the SDN/OF domains is illustrated. The RESTCONF messages, sent from ABNO to the per-domain controllers, requesting the creation of a METER_BAND with $100 \mathrm{Mbps}$ band-rate and the subsequent flow creation request with the associated METER_ID are shown in detail in the lower part of the capture. The meters are only introduced at the first and last nodes of the E2E connection, limiting the incoming traffic from both endpoints.

Finally, we demonstrate the per-flow meter traffic limitation concept in the data plane by showing a real traffic capture of the throughput reach by three different flows after traversing the OFSwitch, with and without attaching a meter band to them (Fig.4a, Fig4.b). The Iperf Bandwidth Measurement Tool has been employed to obtain the results placing a client into the machine connected to the input port and a server measuring the bandwidth achieved into a second machine, connected at the output port of the switch. The three meters are set to 100, 300 and 600 Mbps, we can observe how after applying the method previously described differentiated QoS levels to each flow have been successfully achieved.

\section{Conclusions}

SDN Orchestration architecture of Multilayer TEaware E2E services is presented and experimentally validated. E2E QoS is enabled by the proposed architecture and automatic Multilayer Traffic Aggregation demonstrated by the introduction of a novel the per-flow meter traffic limitation technique which has been shown

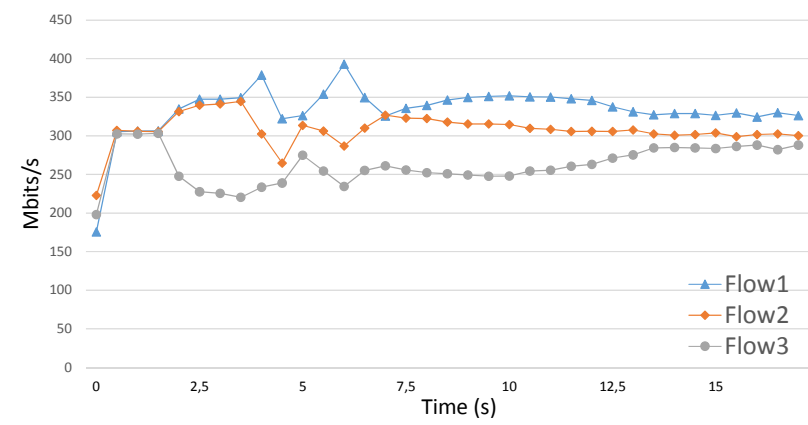

a)

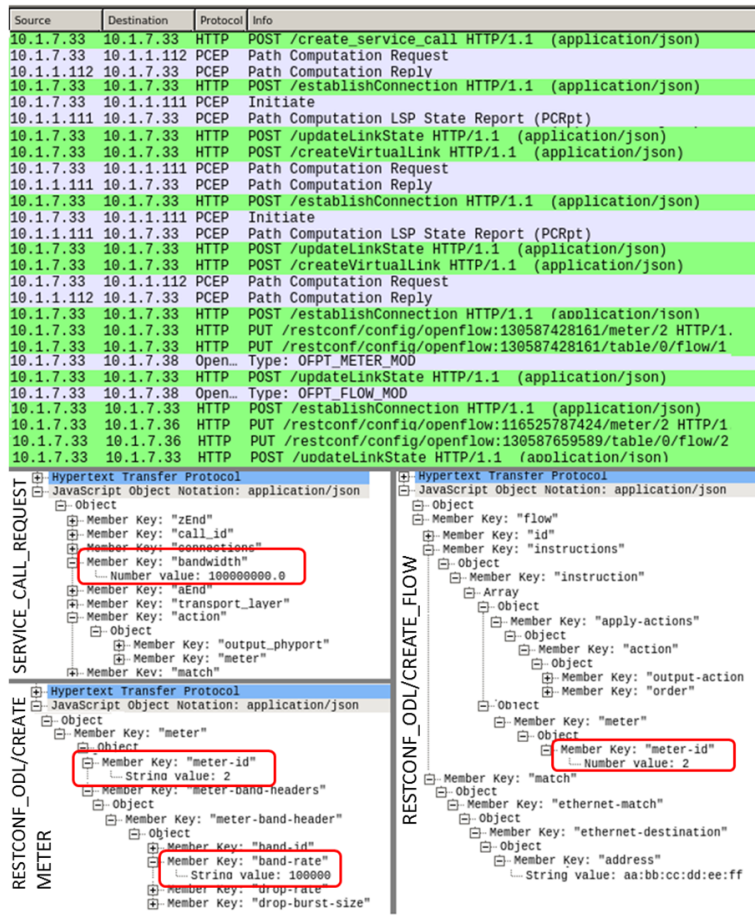

Fig. 3: Multilayer E2E TE-aware service orchestration Wireshark capture.

effectively in a real test field.

\section{Acknowledgements}

EC's FP7 (IP IDEALIST project - 317999), and Spanish MINECO project FARO (TEC201238119)

\section{References}

[1] ONF, "Open Networking Forum: OpenFlow Switch Specifcation", ONF v.1.3.0, June, 2012.

[2] D.King, et al.,"A PCE-based Architecture for Applicationbased Network Operations", IETF draft, 2014.

[3] R. Casellas, et al.,"Overarching Control of Flexi Grid Optical Networks: Interworking of GMPLS and OpenFlow Domains", J. Lightwave Technol., Vol. 33, No. 6, pp. 1-9, (2015).

[4] A.Mayoral et al.,"Experimental Seamless Virtual Machine Migration Using a SDN IT and Network Orchestrator" OFC 2015, Th2A.40-1.

[5] R.Martinez et al., "Experimental Evaluation of DelaySensitive Traffic Routing in Multi-Layer (Packet-Optical) Aggregation Networks for Fixed Mobile Convergence", ECOC, We.3.E.3, London (2013).

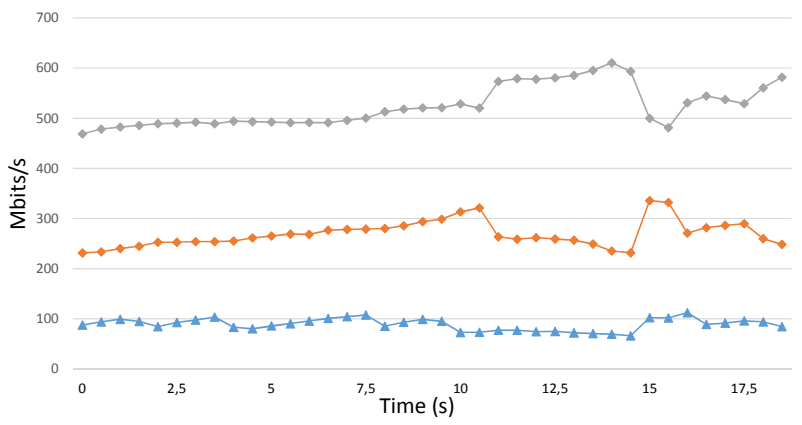

b)

Fig. 4: Per-flow bandwidth limitation; a) IO graph OF Switch output_port without meter limitation; b) IO graph OF Switch output_port throughput with 600, 300 and 100 Mbps meter limitation. 